\title{
Cerebral Blood Flow in Glaucoma Patients
}

\author{
Alon Harris, MS, PhD, FARVO ${ }^{\star}$, , Brent Siesky, PhD ${ }^{\ddagger}$, and Barbara Wirostko, MD $\S$ \\ "Department of Ophthalmology, Indiana University Medical Center, Indianapolis, IN \\ tDepartment of Cellular and Integrative Physiology, Clinical Research, Indiana University Medical \\ Center, Indianapolis, IN \\ ¥Ocular Vascular Research Center, Vascular Reading Center, Glaucoma Research and \\ Diagnostic Center, Glick Eye Institute, Indiana University Medical Center, Indianapolis, IN \\ $\S$ Department of Ophthalmology, University of Utah School of Medicine, Salt lake City, UT
}

\begin{abstract}
Glaucomatous damage has been described as a slowly progressive neuronal degenerative process along the visual pathway. Decreased cerebral and ocular blood flow as well as impaired vascular autoregulation has been identified in glaucoma and have been shown to correlate with visual field loss. In low-tension glaucoma patients, diffuse cerebral ischemic changes have been detected through magnetic resonance imaging. Given these findings, it seems that for some patients, glaucomatous damage may be the ocular manifestation of a more widespread vascular abnormality involving the brain rather than a separate process isolated only to the eye and its immediate vasculature.
\end{abstract}

Within the central visual pathway, the majority of retinal ganglion cells (RGC) extend axons through the optic nerve, cross at the chiasm, and follow as optic tracts into the cerebral cortex to terminate in the lateral geniculate nucleus (LGN) in the thalamus. From here neuronal projections extend to the primary visual cortex in the occipital lobe via the optic radiations.

The blood supply to the central visual system is derived from branches of the internal carotid artery (ICA), including the ophthalmic artery (OA), anterior choroidal artery, anterior cerebral artery (ACA), and middle cerebral artery (MCA). The posterior cerebral arteries (PCA) arise off the vertebrobasilar arteries. The OA is the main blood supply to the orbit and gives rise to ciliary arteries that supply the choroid and optic nerve head and the central retinal artery that supplies the retina. The ACA goes above the optic chiasm to the medial surface of the cerebral hemispheres while the MCA provides blood to the entire lateral aspect of each hemisphere. The anterior choroidal artery runs posteriorly with the optic tracts and supplies the optic tracts, LGN and optic radiations. The lateral temporal and occipital lobes are primarily supplied by the PCA off of the vertebrobasilar system where

Copyright () 2013 by Lippincott Williams \& Wilkins Disclosure: The authors declare no conflict of interest. 
changes in blood flow create classic visual field defects, e.g., hemianopsia and visual agnosia.

It is important to realize that the blood supply to the globe is dependent upon these branches, the short posterior ciliary and central retinal artery branching off the OA. The short posterior ciliary arteries in particular supply the choroid and optic nerve head and are vulnerable to changes in systemic blood pressure, perfusion pressure and vascular dysregulation. Doppler imaging has demonstrated increased blood flow resistance and decreased blood flow velocities in the OA and PCAs of patients with primary open angle glaucoma (POAG), exfoliative glaucoma (XFG) and normal tension glaucoma (NTG) thus suggesting a role that changes in blood supply can contribute and or correlate with optic nerve head pathology. ${ }^{1,2}$

\section{GLAUCOMA AND DEGENERATIVE CHANGES WITHIN THE CENTRAL VISUAL PATHWAY}

In glaucoma, structural RGC loss such as thinning of the retinal nerve fiber layer and optic nerve head 'cupping' can be observed and quantified. Glaucomatous structural damage has been described further along the central visual pathway, with atrophic changes observed in the LGN and visual cortex in experimental primate glaucoma models as well as in a human case study. Functional loss manifesting as visual field deficits have been correlated with structural neural degenerative changes in the central visual pathway. ${ }^{3-6}$ Recently, magnetic resonance imaging (MRI) of the LGN has been performed in human glaucoma patients in an attempt to link LGN degenerative changes seen in these previous primate studies to those observed through human LGN neuroimaging in vivo. ${ }^{7}$ However, given the small sample sizes it is difficult to determine whether the decreased LGN size visualized in glaucoma patients is a meaningful measurement of degenerative change or attributable to other disease processes, aging and or human variation, thus further studies are needed.

\section{VASCULAR INSUFFICIENCY IN GLAUCOMA}

In addition to degeneration within the central visual pathway, MRI has demonstrated a higher prevalence of cerebral white matter lesions and small vessel ischemic changes in both XFG and NTG when compared to controls. ${ }^{8,9}$ Corpus callosum atrophy has been identified via MRI in NTG patients in conjunction with cerebral infarcts. ${ }^{10}$ These findings along with evidence for coexisting vascular risk factors in the setting of glaucomatous optic nerve damage may suggest that ischemia is a contributor to glaucoma progression. ${ }^{9,11,12}$ It is unclear whether altered blood flow in the progression of glaucoma results from a localized retrobulbar vascular insufficiency or a more extensive central neurologic process involving the entire cerebral circulation. ${ }^{13}$

Transcranial Doppler (TCD) measurements of MCA mean and peak systolic blood flow velocities provide evidence for cerebral vascular insufficiency in the setting of glaucoma. Harris et al. has described decreased MCA mean and peak systolic blood flow velocities as well as impaired autoregulation in POAG patients versus normal controls (Fig. 1). ${ }^{14}$ Additionally, such alterations in cerebral blood flow have also been identified in other types of glaucoma, with reduced MCA blood flow velocity measurements and impaired cerebral 
autoregulation observed in the setting of both NTG and XFG when compared to healthy controls. ${ }^{15,16}$

\section{DECREASED CEREBRAL BLOOD FLOW CORRELATES WITH GLAUCOMATOUS DAMAGE}

Decreased cerebral blood flow has been associated with glaucomatous damage and visual field deficits. NTG patients with an Alzheimer's Disease-like (AD) perfusion pattern on single photon emission computed tomography scan were found to have more rapidly progressing visual field defects than NTG patients with normal cerebral perfusion patterns. ${ }^{17}$ Furthermore, recent research has shown that early structural changes such as loss of nerve fiber layer tissue in the retina and optic nerve may be an early biomarker of AD. These deficits in the nerve fiber layer of the retina and optic nerve may be the earliest sign of AD. ${ }^{18}$ Thus 'glaucoma like' changes to the optic nerve may occur earlier in AD before damage to the hippocampal region that impacts memory. Patients with NTG and characteristic ischemic changes on brain MRI were found to have deeper depression in the inferior pericentral visual field as compared to those with NTG and nonischemic brain MRIs. ${ }^{19}$

Focal cone electroretinography (ERG) is a useful tool for quantifying central visual function in the setting of POAG because it examines photoreceptors and bipolar cells. These cells should be unaffected by glaucomatous damage within the inner retina and optic nerve, and variations in ERG amplitude may detect ischemic damage to components of the outer retina which are supplied by the ciliary arteries and choroid. ${ }^{20,21}$ In patients with POAG, measurements of MCA mean flow velocity by TCD demonstrated significant correlation with ERG amplitude, mean sensitivity, mean defect, and contrast sensitivity loss. ${ }^{13}$ This study by Harris et al. suggested that reductions in cerebral blood flow are associated with decreased ERG amplitude. ${ }^{13}$

\section{CONCLUSION}

Data taken from various prospective studies suggest that glaucoma may represent 'a sick eye in a sick body.' We propose that, in certain patients, glaucomatous damage may be a specific organ manifestation of global vascular insufficiency and dysfunction rather than a separate disease process isolated only to the eye and its immediate vasculature. This may be especially true in glaucoma patients of African descent who experience both disproportionate systemic vascular complications and glaucoma pathology. A recent analysis found significantly lower blood flow values were identified in all retrobulbar blood vessels in African descent compared to European descent glaucoma patients (Siesky B, Harris A. Differences in ocular blood flow in glaucoma between patients of African and European descent. Paper submitted to J Glaucoma). Although the findings discussed here do not establish a causative relationship between decreased cerebral blood flow and progression of visual field deficits, they do provide support for this hypothesis and warrant further investigation. Understanding the relationship between decreased cerebral blood flow and glaucoma may be relevant in the development of new screening techniques and treatment strategies to preserve vision in glaucoma patients. 


\section{Acknowledgments}

Supported by NIH grant 1R21EY022101-01A1.

\section{References}

1. Martinez A, Sanchez M. Ocular blood flow in glaucoma. Br J Ophthalmol. 2008; 92:301-1302. [PubMed: 18303150]

2. Martinez A, Sanchez M. Ocular haemodynamics in pseudoexfoliative and primary open-angle glaucoma. Eye (Lond). 2008; 22:515-520. [PubMed: 17173007]

3. Yücel YH, Zhang Q, Gupta N, et al. Loss of neurons in magnocellular and parvocellular layers of the lateral geniculate nucleus in glaucoma. Arch Ophthalmol. 2000; 118:378-384. [PubMed: 10721961]

4. Yücel YH, Zhang Q, Weinreb RN, et al. Atrophy of relay neurons in magno- and parvocellular layers in the lateral geniculate nucleus in experimental glaucoma. Invest Ophthalmol Vis Sci. 2001; 42:3216-3222. [PubMed: 11726625]

5. Weber AJ, Chen H, Hubbard WC, et al. Experimental glaucoma and cell size, density, and number in the primate lateral geniculate nucleus. Invest Ophthalmol Vis Sci. 2000; 41:1370-1379.

[PubMed: 10798652]

6. Gupta N, Ang LC, Noël de Tilly L, et al. Human glaucoma and neural degeneration in intracranial optic nerve, lateral geniculate nucleus, and visual cortex. Br J Ophthalmol. 2006; 90:674-678. [PubMed: 16464969]

7. Gupta N, Greenberg G, de Tilly LN, et al. Atrophy of the lateral geniculate nucleus in human glaucoma detected by magnetic resonance imaging. Br J Ophthalmol. 2009; 93:56-60. [PubMed: 18697810]

8. Yüksel N, Anik Y, Altintaş O, et al. Magnetic resonance imaging of the brain in patients with pseudoexfoliation syndrome and glaucoma. Ophthalmologica. 2006; 220:125-130. [PubMed: 16491036]

9. Stroman GA, Stewart WC, Golnik KC, et al. Magnetic resonance imaging in patients with low tension glaucoma. Arch Ophthalmol. 1995; 113:168-172. [PubMed: 7864748]

10. Ong K, Farinelli A, Billson F, et al. Comparative study of brain magnetic resonance imaging findings in patients with low-tension glaucoma and control subjects. Ophthalmology. 1995; 102:1632-1638. [PubMed: 9098255]

11. Leske MC, Heijl A, Hyman L, et al. Predictors of long-term progression in the early manifest glaucoma trial. Ophthalmology. 2007; 114:1965-1972. [PubMed: 17628686]

12. Leske MC, Wu SY, Hennis A, et al. Risk factors for incident open-angle glaucoma: the Barbados Eye Studies. Ophthalmology. 2008; 115:85-93. [PubMed: 17629563]

13. Harris A, Siesky B, Zarfati D, et al. Relationship of cerebral blood flow and central visual function in primary open-angle glaucoma. J Glaucoma. 2007; 16:159-163. [PubMed: 17224767]

14. Harris A, Zarfati D, Zalish M, et al. Reduced cerebrovascular blood flow velocities and vasoreactivity in open-angle glaucoma. Am J Ophthalmol. 2003; 135:144-147. [PubMed: 12566016]

15. Tutaj M, Brown CM, Brys M, et al. Dynamic cerebral autoregulation is impaired in glaucoma. J Neurol Sci. 2004; 220:49-54. [PubMed: 15140605]

16. Akarsu C, Unal B. Cerebral haemodynamics in patients with pseudoexfoliation glaucoma. Eye. 2005; 19:1297-1300. [PubMed: 15650760]

17. Sugiyama T, Utsunomiya K, Ota $\mathrm{H}$, et al. Comparative study of cerebral blood flow in patients with normal-tension glaucoma and control subjects. Am J Ophthalmol. 2006; 141:394-396. [PubMed: 16458708]

18. Valenti DA. Alzheimer's disease and glaucoma: imaging the biomarkers of neurodegenerative disease. Int J Alzheimers Dis. 2011; 2010:793931. [PubMed: 21253485]

19. Suzuki J, Tomidokoro A, Araie M, et al. Visual field damage in normal-tension glaucoma patients with or without ischemic changes in cerebral magnetic resonance imaging. Jpn J Ophthalmol. 2004; 48:340-344. [PubMed: 15295658] 
20. Nork TM, Ver Hoeve JN, Poulsen GL, et al. Swelling and loss of photoreceptors in chronic human and experimental glaucomas. Arch Ophthalmol. 2000; 118:235. [PubMed: 10676789]

21. Block F, Schwarz M. The b-wave of the electroretinogram as an index of retinal ischemia. Gen Pharmacol. 1998; 30:281-287. [PubMed: 9510075] 


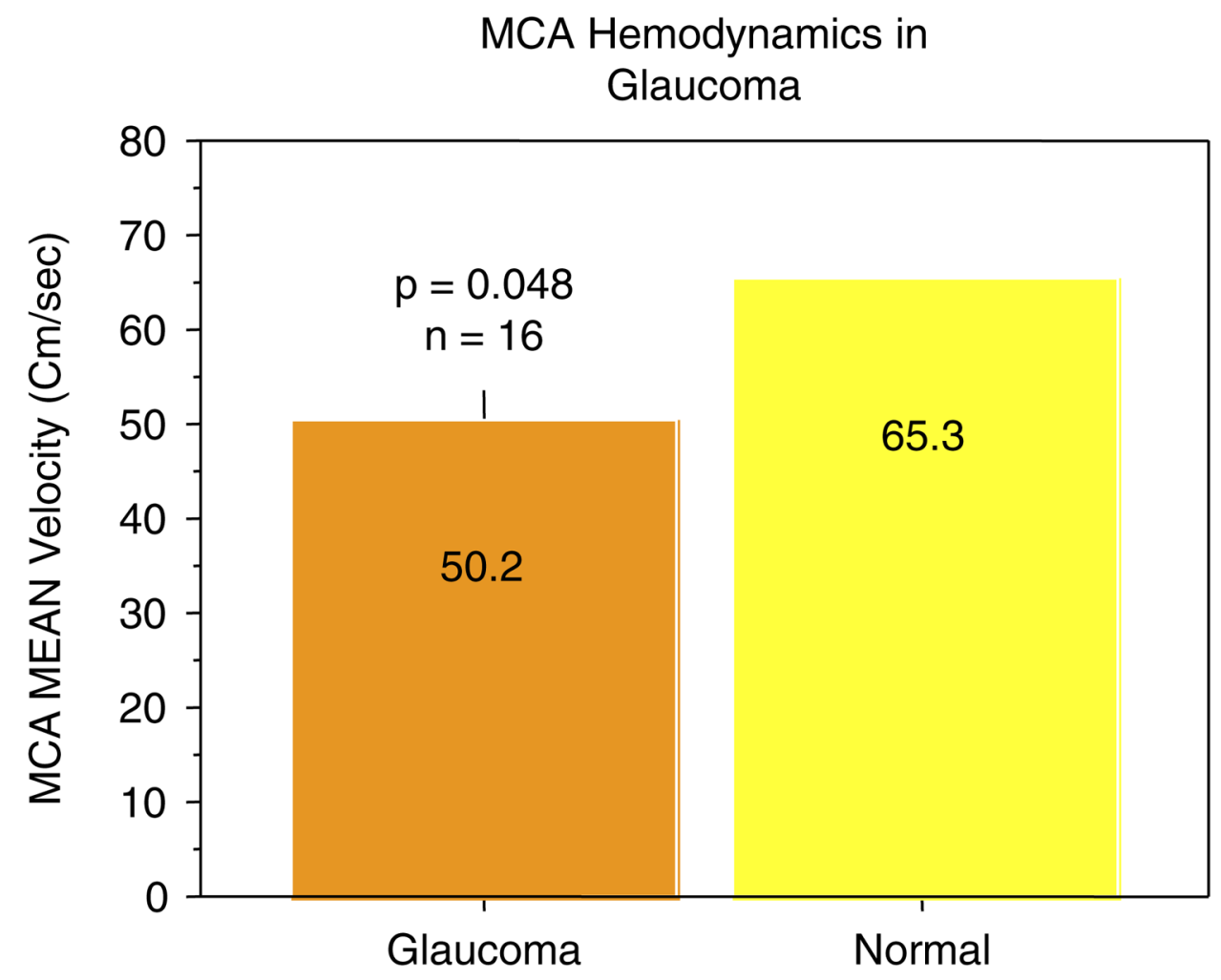

FIGURE 1.

Mean blood flow velocities in the middle cerebral artery (MCA) in patients with glaucoma and in those with normal eyes. MCA mean velocity was statistically significantly lower in glaucoma patients than in controls. Adapted from Harris A, Zarfati D, Zalish M, et al. Am J Ophthalmol 2003;135:144-147. 